\title{
Electron Microscopy of Perokskite Phase Distribution on Light Emitting Edges
}

Hector Calderon ${ }^{1}$, Jiming Bao ${ }^{2}$, Viktor Hadjiev ${ }^{2}$, Zhiming Wang ${ }^{3}$ and Zhaojun Qin ${ }^{2}$

${ }^{1}$ Instituto Politecnico Nacion ESFM, Mexico, Distrito Federal, Mexico, ${ }^{2}$ University of Houston, Houston, Texas, United States, ${ }^{3}$ University of Electronic Science and Technology of China, Chengdu, Sichuan, China (People's Republic)

New and different properties are found at edges of two-dimensional halide perovskites. The source of such properties has been sometimes difficult to find. For example there has been a contoversy about the optical properties of $2 \mathrm{D} \mathrm{CsPb}_{2} \mathrm{Br}_{5}$ and its ion exchanged halides. Recently the root cause of such a behavior has been revealed [1] by using a combination of techniques (Raman and Photoluminiscence, PL), one statitc and the other dynamic with variable hydrostatic pressure. It has been posible to correlate PL with the structure and distinguish different mechanisms of PL from point defect versus extended structures. In that case, it is shown that brigth green emission is due to the presence of $\mathrm{CsPbBr} 3$ nanocrystals overgrown on the surfaces and at the edges of $\mathrm{CsPb}_{2} \mathrm{Br}_{5}$ platelets, producing a bright edge emission. Electron microscopy with atomic resolution has been also helpful in that case to show the structure of the involved phases clearly. This work is useful to open up new opportunities to understand, design and engineer other inorganic perovskites for novel optoelectronic devices. In the present electron microscopy investigation, the involved material is an exfoliated 2D halide perovskite containing a variety of thin crystals. It is of relevance to identify the phases as a function of position, starting from the edge of the material so that the above describe technique can be applied. The main objective is to obtain insigths on the origin of the edge states in 2D perovskytes so that their optical and electrical properties can be controlled. In this investigation the sample is produced to have the $\mathrm{BA}_{2} \mathrm{MA}_{2} \mathrm{~Pb}_{3} \mathrm{Br}_{10}$ perovskite and it shows properties of edge emission. The main objective is to clarify such a property.

The electron microscope work shows a phase distribution with two main components in the exfoliated sample. The edge of the sample is dominated by the $\mathrm{MAPbBr} 3$ perovskite while the interior is $\mathrm{BA}_{2} \mathrm{MA}_{2} \mathrm{~Pb}_{3} \mathrm{Br}_{10}$. The former is obtained by loosing BA. Low dose electrón microscopy in transmission mode has been performed to preserve the genuine phase structure, especially in the presence of water, N, $\mathrm{C}$ and $\mathrm{O}$. Figure 1 shows phase images of nanoparticles that can be found near the edge of the sample. The crystalline structure coincides with the expected projection of the [111] zone axis in the $\mathrm{MAPbBr}_{3}$ perovskyte (sg 221). Figure 2 shows a representative phase image along a zone axis near [104] in the $\mathrm{BA}_{2} \mathrm{MA}_{2} \mathrm{~Pb}_{3} \mathrm{Br}_{10}$ structure ( $\mathrm{sg} 4$ ). The sample is particularly unstable and only 10-15 images can be taken at rather low dose ( $\left.10 \mathrm{e}^{-} / \mathrm{A}^{2} \mathrm{~s}\right)$ and $80 \mathrm{keV}$ in the TEAM05 electron microscope of the NCEM-MF. These focal series are used to produce the reported phase images. They are obtained by means of an exit wave reconstruction procedure [2]. 

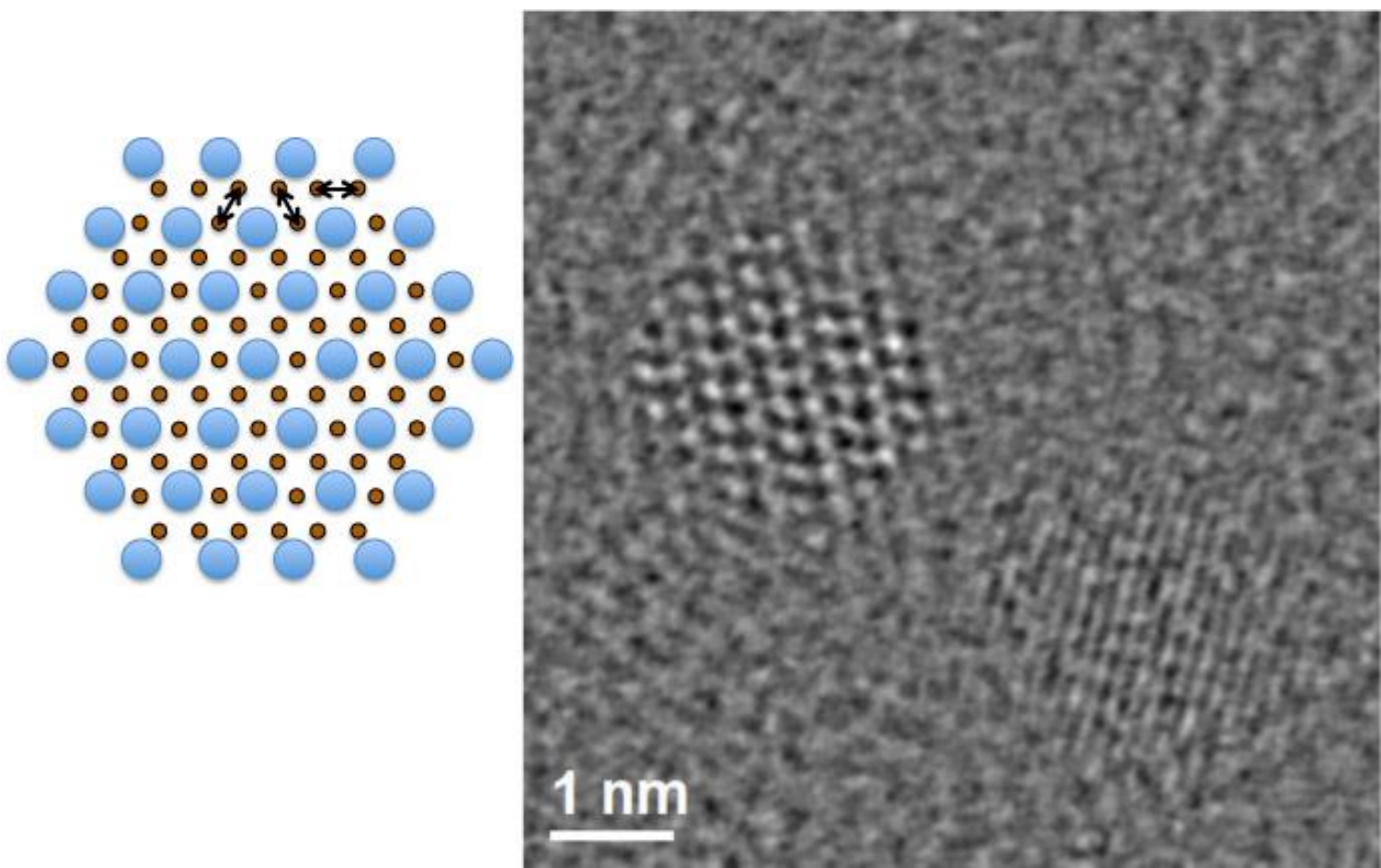

Figure 1. Phase image of nanoparticles at the edge of exfoliated sample. They can be identifiied to the phase MAPbBr3. Zone axis [111]

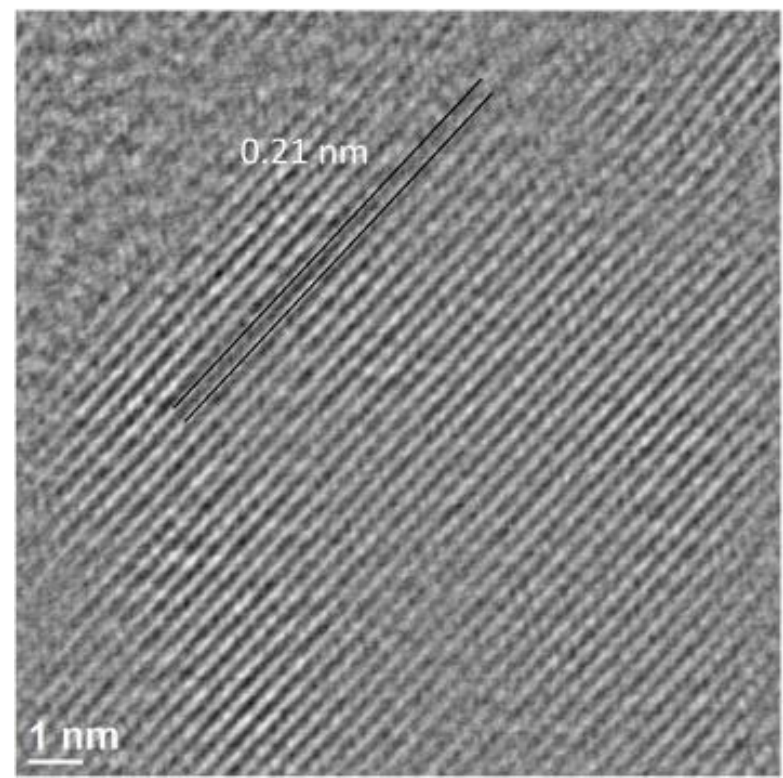

$0.21 \mathrm{~nm}$

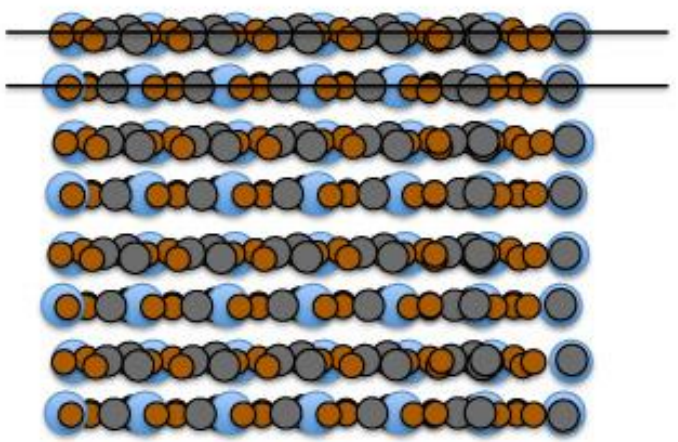

Figure 2. Phase image of $\mathrm{BA} 2 \mathrm{MA} 2 \mathrm{~Pb} 3 \mathrm{Br} 10$ perovskyte as identified in the zone axis [104]

References

1. Extrinsic Green Photoluminiscence from the Edges of 2D Cesium Lead Halides. C. Wang et. al. Advanced Materials 2019,1902492.

2. Work at the Molecular Foundry was supported by the Office of Science, Office of Basic Energy Sciences, of the U.S. Department of Energy under Contract No. DE-AC02-05CH11231. 